\title{
Diversity and Connectivity of Layer 5 Somatostatin-Expressing Interneurons in the Mouse Barrel Cortex
}

\author{
Maximiliano José Nigro, ${ }^{1}$ Yoshiko Hashikawa-Yamasaki, ${ }^{1}$ and $₫$ Bernardo Rudy ${ }^{1,2}$ \\ ${ }^{1}$ Neuroscience Institute and Department of Neuroscience and Physiology, and ${ }^{2}$ Department of Anesthesiology, Perioperative Care and Pain Medicine, \\ New York University School of Medicine, New York 10016
}

Inhibitory interneurons represent $10-15 \%$ of the neurons in the somatosensory cortex, and their activity powerfully shapes sensory processing. Three major groups of GABAergic interneurons have been defined according to developmental, molecular, morphological, electrophysiological, and synaptic features. Dendritic-targeting somatostatin-expressing interneurons (SST-INs) have been shown to display diverse morphological, electrophysiological, and molecular properties and activity patterns in vivo. However, the correlation between these properties and SST-IN subtype is unclear. In this study, we aimed to correlate the morphological diversity of layer 5 (L5) SST-INs with their electrophysiological and molecular diversity in mice of either sex. Our morphological analysis demonstrated the existence of three subtypes of L5 SST-INs with distinct electrophysiological properties: T-shaped Martinotti cells innervate L1, and are low-threshold spiking; fanning-out Martinotti cells innervate L2/3 and the lower half of L1, and show adapting firing patterns; nonMartinotti cells innervate L4, and show a quasi-fast spiking firing pattern. We estimated the proportion of each subtype in L5 and found that T-shaped Martinotti, fanning-out Martinotti, and Non-Martinotti cells represent $\sim 10, \sim 50$, and $\sim 40 \%$ of L5 SST-INs, respectively. Last, we examined the connectivity between the three SST-IN subtypes and L5 pyramidal cells (PCs). We found that L5 T-shaped Martinotti cells inhibit the L1 apical tuft of nearby PCs; L5 fanning-out Martinotti cells also inhibit nearby PCs but they target the dendrite mainly in L2/3. On the other hand, non-Martinotti cells inhibit the dendrites of L4 neurons while avoiding L5 PCs. Our data suggest that morphologically distinct SST-INs gate different excitatory inputs in the barrel cortex.

Key words: barrel cortex; cortical circuits; inhibition; interneurons; somatostatin

\section{Significance Statement}

Morphologically diverse layer 5 SST-INs show different patterns of activity in behaving animals. However, little is known about the abundance and connectivity of each morphological type and the correlation between morphological subtype and spiking properties. We demonstrate a correlation between the morphological and electrophysiological diversity of layer 5 SST-INs. Based on these findings we built a classifier to infer the abundance of each morphological subtype. Last, using paired recordings combined with morphological analysis, we investigated the connectivity of each morphological subtype. Our data suggest that, by targeting different cell types and cellular compartments, morphologically diverse SST-INs might gate different excitatory inputs in the mouse barrel cortex.

\section{Introduction}

GABAergic interneurons represent $\sim 15 \%$ of the neuronal population in the mouse barrel cortex and their wide diversity allows

\footnotetext{
Received Aug. 24, 2017; revised Nov. 5, 2017; accepted Dec. 2, 2017.

Author contributions: M.J.N. and B.R. designed research; M.J.N. and Y.H.-Y. performed research; M.J.N. and Y.H.-Y. analyzed data; M.J.N. and B.R. wrote the paper.

This work was supported by NIH Grants NS30989; R21NS093987 and P01NS074972 to B.R. We thank all the members of the Rudy laboratory and Dr. G. Quattrocolo for helpful comments on the paper.

The authors declare no competing financial interests.

Correspondence should be addressed to Dr. Bernardo Rudy, 522 First Avenue, Smilow Research Building, Sixth Floor, New York, NY 10016. E-mail: Bernardo.rudy@nyumc.org.

M.J. Nigro's present address: Kavli Institute for System Neuroscience, NTNU Faculty of Medicine, Postboks 8905, 7491 Trondheim, Norway.
}

them to exert a tight control over computations performed by excitatory neurons (Tremblay et al., 2016). Dendritic targeting GABAergic interneurons expressing the neuropeptide somatostatin (SST-INs) represent $\sim 30 \%$ of GABAergic interneurons (Xu et al., 2010; Tremblay et al., 2016). SST-INs are a very diverse group of inhibitory cells that show differential expression of molecular markers, different morphologies and electrophysiological properties (Wang et al., 2004; Ma et al., 2006). Of particular interest are morphological differences suggesting functional specializations. SST-INs include the Martinotti cells, neurons pres- 
ent in supragranular and infragranular layers and characterized by an ascending axon that reaches and branches in layer 1 (L1; Martinotti, 1889; Ramon y Cajal, 1891; Wang et al., 2004). On the other hand, Non-Martinotti cells have been described in L4 and $5 \mathrm{~b}$ of the mouse barrel cortex and specifically innervate L4 and lack an ascending axon reaching L1 (Ma et al., 2006; Xu et al., 2013). Both morphological types have been recently described in vivo in L5 of the barrel cortex (Muñoz et al., 2014, 2017). Muñoz et al. (2017) showed that the morphological identity of the SSTINs correlates with their in vivo activity pattern during active wakefulness. Moreover, based on the morphological features of the axon of the Martinotti cells this study distinguished two types of Martinotti cells with distinct in vivo activity patterns: fanningout Martinotti cells with a broad axonal domain in L2/3, were activated during whisking periods; whereas cells with most of their axon branching in L1, called T-shaped Martinotti cells, were inhibited during whisking transitions (Muñoz et al., 2017).

L5 SST-INs also show diverse firing properties when depolarized by current injection (Halabisky et al., 2006; Ma et al., 2006; Tremblay et al., 2016). However, the relationship between the morphological and electrophysiological diversity remains poorly understood. Furthermore, different populations of L5 SST-INs express several molecular markers. These include calretinin, calbindin, reelin, and neuropeptide $\mathrm{Y}$; however, the functional significance of this molecular diversity is still unclear (Wang et al., 2004; Ma et al., 2006). Understanding of the correlation between morphological, electrophysiological and molecular diversity can provide experimental means to target and manipulate specific types of SST-INs.

Several studies have highlighted the importance of SST-INs in cortical computations, and the morphological diversity is likely to be functionally significant since distinct SST-INs subtypes have axons with very different layer distributions. However, little is known about the specific postsynaptic partners of different morphological subtypes of L5 SST-INs. For instance, it has been reported that L5 Martinotti cells provide dendritic feedback inhibition to L5 pyramidal cells (PCs), but it is unknown whether both types of Martinotti cells are connected to L5 PCs and participate in this type of inhibition (Silberberg and Markram, 2007). Moreover, the output connectivity of non-Martinotti cells has not been studied. These cells could provide dendritic inhibition of L5 PCs by synapsing on their apical dendrite as it crosses L4 and/or target local L4 neurons. Knowledge of the connectivity of specific SST-INs is essential to understand the functional significance of SST-IN diversity.

In the present study we used slice electrophysiology to study how the morphological diversity of SST-INs correlates with their electrophysiological diversity. We focused on L5, the main output layer of the cortex, and the layer where SST-INs are most abundant and diverse. We use this knowledge to infer the relative proportion of each morphological subtype in the total population of L5 SST-INs. To address the correlation of morphological, electrophysiological, and molecular features we used intersectional genetics (He et al., 2016). We found that intersectional genetics can be used to obtain mouse lines where the expression of fluorescent proteins in specific morphological types is enriched. Moreover, we used paired recordings to characterize the connectivity between L5 SST-INs and L5 PCs. These studies showed that Martinotti and non-Martinotti cells belong to distinct inhibitory circuits within L5.

\section{Materials and Methods}

Animals. All experimental procedures were conducted in accordance with the National Institute of Health guidelines and were approved by the Institutional Animal Care and Use Committee of the NYU School of Medicine. Mice used in this study were bred at the animal facility of the Department of Physiology. To target somatostatin neurons we crossed the Somatostatin-IRES-CRE line (https://www.jax.org/strain/028864; RRID:IMSR_JAX:028864) with the Ai9 td-Tomato reporter mouse (https://www.jax.org/strain/007909; RRID:IMSR_JAX:007909) or with a YFP reporter (https://www.jax.org/strain/006148; RRID:IMSR_JAX: 006148). To obtain the intersectional mice we first crossed the SomatostatinIRES-FlpO (https://www.jax.org/strain/028579; RRID:IMSR_JAX:028579) with either Calretinin-IRES-Cre (https://www.jax.org/strain/010774; RRID:IMSR_JAX:010774) or Calb1-IRES-Cre (https://www.jax.org/ strain/028532; RRID:IMSR_JAX:028532). Double-transgenic animals were then crossed with the td-Tomato intersectional reporter Ai65 (https://www.jax.org/strain/021875; RRID:IMSR_JAX:021875). Mice of either sex were used.

Immunohistochemistry. Animals were killed with intraperitoneal injection of sodium pentobarbital ( $100 \mathrm{mg} / \mathrm{Kg}$ body weight), and intracardially perfused with PBS followed by PFA 4\%. The brain was postfixated for $1 \mathrm{~h}$ in PFA 4\%. After washing out the PFA, the brain was glued onto the stage of a vibratome (Leica) and $70 \mu \mathrm{m}$ slices of the barrel cortex were cut in cold PBS. Slices were permeabilized for $1 \mathrm{~h}$ in PB and Triton (1\%) at room temperature. After permeabilization, the slices were incubated $1 \mathrm{~h}$ in blocking solution: NGS $10 \%$, BSA $1 \%$, gelatin $0.2 \%$, Triton $0.5 \%$ in PBS. Primary antibodies were incubated at $4^{\circ} \mathrm{C}$ for $48 \mathrm{~h}$, and washed out in PBS and Triton $0.2 \%$. Secondary antibodies were incubated overnight at $4^{\circ} \mathrm{C}$. Fluorescent Nissl (Neurotrace, ThermoFisher) staining was performed before mounting. We used the following antibodies: rabbit anti-SST (1:500; Peninsula; RRID:AB_518614), rabbit anti-Calb (1:2000; Swant; RRID:AB_10000340), mouse anti-Calb (1:2000; Swant; RRID: AB_10000347), rabbit anti-CR (1:1000; Swant), and chicken anti-GFP (1:1000; Abcam; RRID:AB_300798).

Preparation of brain slices. Mice of either sex (P20-P40) were killed with intraperitoneal injection of Sodium Pentobarbital ( $100 \mathrm{mg} / \mathrm{Kg}$ body weight), and intracardially perfused with refrigerated cutting solution of the following composition (in $\mathrm{mm}$ ): $93 \mathrm{~N}$-methyl-D-glucamine, $3 \mathrm{KCl}$, $1.25 \mathrm{NaH}_{2} \mathrm{PO}_{4}, 30 \mathrm{NaHCO}_{3}, 20$ HEPES, 10 glucose, $5 \mathrm{MgCl}_{2}, 0.5 \mathrm{CaCl}_{2}$, $5 \mathrm{~N}$-acetylcysteine, saturated with $\mathrm{O}_{2} 95 \% / \mathrm{CO}_{2} 5 \%$. After decapitation, the posterior part of the brain was removed with an angle of $\sim 20^{\circ}$ from the coronal plane in the rostrocaudal direction. This angle of slicing increased the chance to preserve the ascending axon of SST-INs, and allowed us to record from the whole rostrocaudal extent of the barrel cortex. The part of the brain containing the barrel field was glued on the stage of a vibratome (VT1200S, Leica) filled with cold cutting solution. Slices $(300 \mu \mathrm{m})$ were transferred to a holding chamber filled with bubbled cutting solution warmed at $35^{\circ} \mathrm{C}$ for $15 \mathrm{~min}$. After the initial recovery the slices were transferred to a holding chamber filled with holding solution of the following composition (in $\mathrm{mm}$ ): $92 \mathrm{NaCl}, 3 \mathrm{KCl}$, $30 \mathrm{NaHCO}_{3}, 1.25 \mathrm{NaH}_{2} \mathrm{PO}_{4}, 20$ HEPES, 10 glucose, $5 \mathrm{MgCl}_{2}, 0.5 \mathrm{CaCl}_{2}$, $5 \mathrm{~N}$-acetylcysteine, saturated with $\mathrm{O}_{2} 95 \% / \mathrm{CO}_{2} 5 \%$. The slices were maintained at room temperature for $1-5 \mathrm{~h}$ before recordings.

Electrophysiological recordings. The slices were transferred to a recording chamber mounted on an upright microscope (Olympus BW50) and perfused with recording solution of the following composition (in $\mathrm{mM}$ ): $124 \mathrm{NaCl}, 3 \mathrm{KCl}, 26 \mathrm{NaHCO}_{3}, 1.25 \mathrm{NaH}_{2} \mathrm{PO}_{4}, 10$ glucose, $1 \mathrm{MgCl}_{2}, 1.6$ $\mathrm{CaCl}_{2}$, saturated with $\mathrm{O}_{2} 95 \% / \mathrm{CO}_{2} 5 \%$. All experiments were performed at $30-32^{\circ} \mathrm{C}$. Characterization of the electrophysiological properties was done in most cases in presence of synaptic blockers: $10 \mu \mathrm{M}$ DNQX-Na $25 \mu \mathrm{M} \mathrm{APV}$, and $10 \mu \mathrm{M}$ gabazine. Healthy td-Tomato-expressing cells were selected for recordings but no other criterion was used to select the cells. Whole-cell patch-clamp recordings were performed with borosilicate pipettes (Sutter Instruments) with a resistance of 3-6 M $\Omega$. For current-clamp experiments, the pipette solution was as follows (in $\mathrm{mM}$ ): $130 \mathrm{~K}$-gluconate, $10 \mathrm{KCl}$, HEPES, 0.2 EGTA, 4 ATP-Mg, 0.3 GTP-Na, 5 phosphocreatine-Tris 2 , $\mathrm{pH}$ 7.3. For voltage-clamp experiments the pipette solution was as follows (in mM): $130 \mathrm{Cs}$-methanesulfonate, $5 \mathrm{CsCl}$, 
10 HEPES, 0.2 EGTA, 4 ATP-Mg, 0.3 GTP-Na, 5 phosphocreatine-Tris ${ }_{2}$, QX314-Cl, $\mathrm{pH}$ 7.3. In all recordings biocytin $0.3-0.5 \%$ was added to the pipette solution. Membrane potentials reported were not corrected for a calculated liquid junction potential of $14 \mathrm{mV}$ (current-clamp) or $10 \mathrm{mV}$ (voltage-clamp). Electrophysiological recordings were performed with a MultiClamp 700B (Molecular Devices), digitized with a 1440 Digitizer (Molecular Devices), interfaced with a personal computer with pClamp 10.3 (Molecular Devices; RRID:SCR_011323). Data were sampled at $10-40 \mathrm{kHz}$, and low-pass filtered at $10 \mathrm{kHz}$. Passive and active membrane properties were measured in current-clamp from a holding potential of $-65 \mathrm{mV}$. The electrophysiological parameters analyzed were defined as follows:

Resting membrane potential $\left(V_{\text {rest }}, \mathrm{mV}\right)$ : membrane potential measured with no current applied $(I=0$ mode);

Input resistance (IR; in $M \Omega$ ): resistance measured from Ohm's law from the peak of voltage responses to hyperpolarizing current injections $(-40$ or $-50 \mathrm{pA})$;

Sag ratio (dimensionless): measured from voltage responses to hyperpolarizing current injections with peaks at $-90 \pm 4 \mathrm{mV}$, as the ratio between the voltage at steady-state and the voltage at the peak;

Action potential threshold $\left(\mathrm{AP}_{\text {thre }}\right.$; in $\left.\mathrm{mV}\right)$ : measured from action potentials (APs) evoked at rheobase with $500 \mathrm{~ms}$ current injections, as the membrane potential where the rise of the AP was $20 \mathrm{mV} / \mathrm{ms}$;

AP half-width (HW; in ms): duration of the AP at half-amplitude from $\mathrm{AP}_{\text {thre; }}$;

$\mathrm{AP}$ maximum rate of rise $\left(\mathrm{AP}_{\text {rise }}\right.$; in $\left.\mathrm{mV} / \mathrm{ms}\right)$ : measured from $\mathrm{APs}$ evoked at rheobase as the maximal voltage slope during the upstroke of the AP;

Afterhyperpolarization (AHP) duration $\left(\mathrm{AHP}_{\text {dur }}\right.$; in $\left.\mathrm{ms}\right)$ : measured from APs evoked at rheobase as the time difference between the peak of the fAHP and the most depolarized membrane potential following the medium AHP (mAHP) in a time window of $200 \mathrm{~ms}$;

AHP shape (in $\mathrm{mV}$ ): measured as the difference between the peak of the afterdepolarization (ADP) and the peak of the mAHP. In cells where a mAHP was not detected the AHP shape was measured between two points in a $10 \mathrm{~ms}$ time window starting $4 \mathrm{~ms}$ after the peak of the fAHP;

Maximal firing frequency $\left(F_{\max }\right.$; in $\left.\mathrm{Hz}\right)$ : maximal firing frequency evoked with 1-s-long depolarizing current steps;

Adaptation (dimensionless): measured from trains of 35 APs as [1 $\left.\left(F_{\text {first }} / F_{\text {last }}\right)\right]$, where $F_{\text {first }}$ and $F_{\text {last }}$ are, respectively, the frequencies of the first and last ISI;

Rebound APs: number of APs elicited in response the end of a 1-s-long hyperpolarizing voltage deflection where the steady-state voltage response was $-90 \mathrm{mV}$.

For dual and triple recordings between SST interneurons and excitatory neurons the postsynaptic elements were recorded in voltage-clamp at a holding potential of $+10 \mathrm{mV}$, in presence of $2 \mathrm{~mm}$ kynurenic acid-Na.

Series resistance $\left(R_{\mathrm{s}}\right)$ in current-clamp was compensated through the bridge balance circuit of the amplifier after pipette capacitance neutralization, and data were discarded if $R_{\mathrm{s}}$ was $>40 \mathrm{M} \Omega$. In voltage-clamp recordings, $R_{\mathrm{s}}$ was compensated to $75-80 \%$, and data were discarded if $R_{\mathrm{s}}$ was $>20 \mathrm{M} \Omega$.

Morphological reconstructions and analysis. In all recordings biocytin $(0.3-0.5 \%)$ was added to the pipette solution. After the recording the slices were fixated in 4\% PFA overnight. After washing out the PFA, the slices were incubated with Alexa-conjugated streptavidin in PBS and Triton-X $0.5 \%$ overnight at room temperature. After washing with PBS the slices were mounted in Fluoromount-G (SouthernBiotech) and stored at $4^{\circ} \mathrm{C}$. Confocal stacks were acquired with a Zeiss LSM800 microscope at $40 \times$ or $63 \times$. Stacks were imported in Neurolucida for tracing. Reconstructions were analyzed in Neurolucida Explorer. The ascending axon is defined here as the axon located in L1-L4. Axonal nodes represent branching points and have been previously used as a measure of axonal targeting (Blasdel and Lund, 1983; Florence and Casagrande, 1987; Freund et al., 1989; Lee et al., 2005; Yamada et al., 2010). Principal component analysis of morphological parameters was performed on the absolute axonal length in L1-L5, absolute number of nodes in L1-L5, relative amount of ascending axon in L1-L4, and relative amount of nodes of ascending axon in L1-L4 (total of 16 morphological parameters). Putative synaptic contacts are defined as appositions between axonal swellings and the dendrites of the postsynaptic cell. Paired recordings were imaged at $63 \times$ with a Zeiss LSM800 confocal microscope and stacks were imported to Neurolucida where appositions were marked.

Experimental design and statistical analysis. Electrophysiological recordings were analyzed in Clampfit (Molecular Devices) and Origin 8.2 (Origin; RRID:SCR_002815). Principal component analysis, hierarchical cluster analysis, and the linear discriminant classifier were performed using the statistics package of MATLAB (MathWorks; RRID:SCR_001622). Statistical analysis was performed in Origin 8.2.

\section{Results}

\section{Morphological diversity of L5 SST interneurons}

To examine the relationship between the morphological diversity of L5 SST-INs and their electrophysiological properties and estimate the representation of each subtype in the total SST-IN population, we performed whole-cell recordings from L5 SST-INs from slices obtained from three transgenic mouse lines: SST-cre, where $95.6 \%$ of td-Tomato cells were found to coexpress SST; SST/CR, to target cells coexpressing SST and calretinin; and SST/ Calb, to target cells coexpressing SST and calbindin. We recently reported that the SST/CR intersection results in the labeling of SST-INs with axons targeting upper layers (He et al., 2016). Here we observed that in the SST/CR intersectional mouse line $\sim 47 \%$ of SST-INs in L $2 / 3,14 \%$ of SST-INs in L5a, and $4 \%$ of SST-INs in L5b express tdTomato (Fig. $1 B, F$ ). In contrast, in the SST/Calb mouse line a larger proportion of SST-INs express tdTomato (Fig. 1C). The proportion of tdTomato-expressing cells in L5b ( $\sim 67 \%$ of SST-INs) was larger than in L5a ( $\sim 44 \%$ of SST-INs; Fig. $1 C, G$ ). In both intersectional lines the majority of tdTomatoexpressing cells coexpressed either CR or Calb (Fig. 1D-G). We measured the overlap of CR and Calb in the SST-cre mouse line by triple immunostainings in the SST-cre/Rosa26YFP line. We found very little $(2.2 \%)$ overlap of CR and Calb in the SST-cre line specially in deep layers (Fig. 2E). However the proportion of YFP cells expressing $\mathrm{CR}$ and Calb was higher in L2/3 (15.9\%; Fig. 2E). The amount of YFP IR cells expressing both CR and Calb over the YFP-CR IR population was $>50 \%$ throughout layers, indicating that approximately half of the cells labeled in the SST-CR line are also labeled in the SST-Calb mouse line (Fig. $2 F$ ).

We morphologically reconstructed and analyzed a total of 30 neurons: 19 in the SST-cre, 5 in the SST/CR, and 6 in the SST/ Calb mouse lines. Inspection of the total axonal length and number of axonal nodes in different layers for each SST-IN allowed us to identify cells that corresponded to each of the three morphological types described in vivo: 8 Non-Martinotti, 13 fanning-out Martinotti, and 9 T-shaped Martinotti cells (Muñoz et al., 2017; Fig. $3 A-C$ ). The ascending axon of each morphological group exhibited a specific layer distribution of relative axonal length and proportion of axonal nodes (Fig. 3D). A principal component analysis of 16 morphological axonal parameters of the reconstructed cells showed that the whole dataset could be divided in three morphological groups in the principal component space (Fig. $3 F$ ). Moreover the first three principal components, accounting for $93 \%$ of the variance, are mostly represented by features of the ascending axon. Because slicing artifacts can affect the interpretation of reconstructions obtained from in vitro recordings we adopted a more quantitative and unsupervised approach to understand the structure of our morphological dataset. First, we selected the best-preserved cells to perform a hierarchical cluster analysis of the features of the ascending axon. For this analysis we used only five putative non-Martinotti cells with at least $3500 \mu \mathrm{m}$ of axon in L4, and 12 Martinotti cells having at least $1000 \mu \mathrm{m}$ of axon in L1. We reasoned that applying a quality 

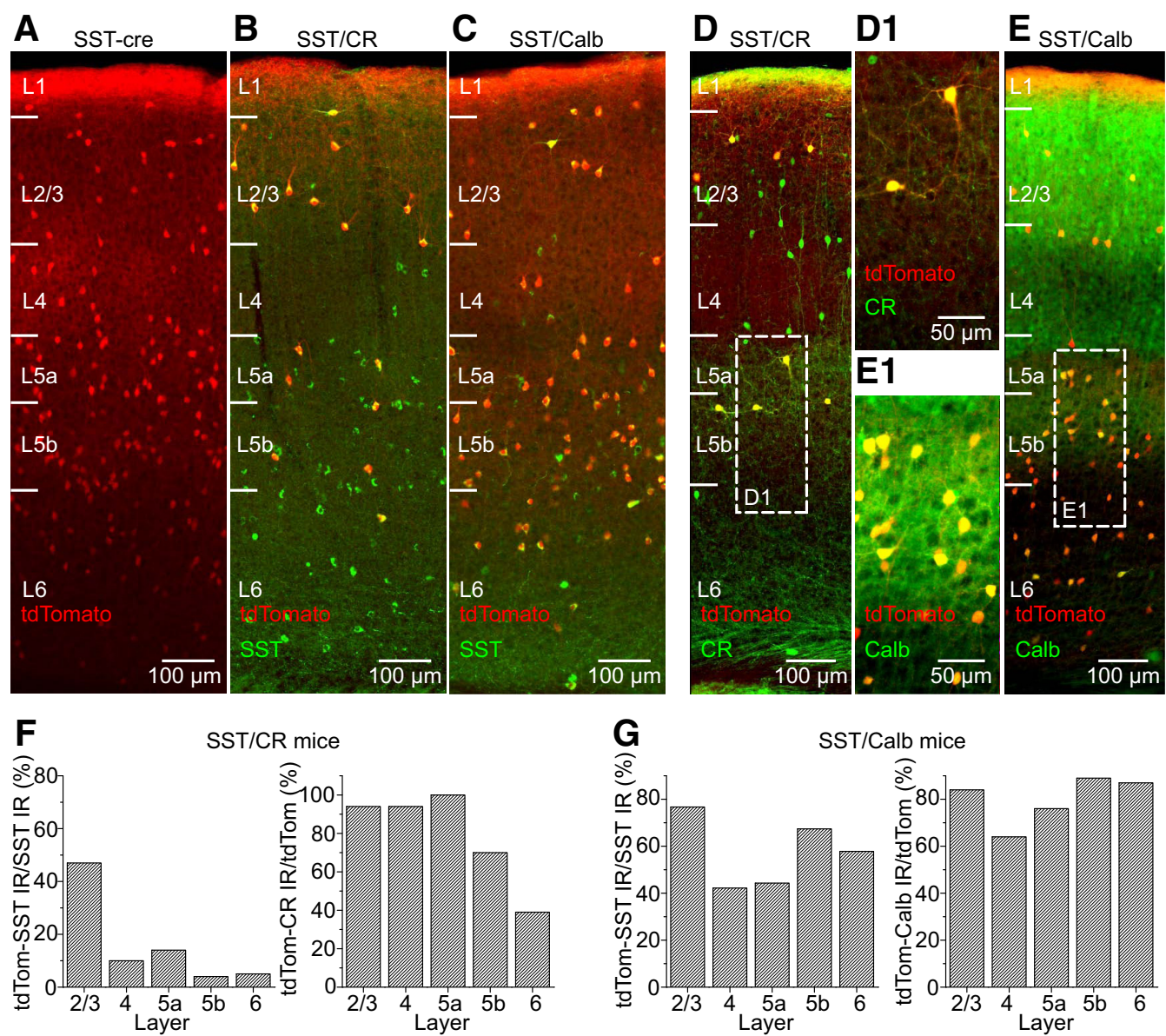

Figure 1. $\boldsymbol{A}-\boldsymbol{C}$, Confocal stacks showing the distribution of td-Tomato-expressing cells in the SST-cre line $(\boldsymbol{A})$, the SST/CR intersectional line $(\boldsymbol{B})$ and the SST/Calb intersectional line $(\boldsymbol{C})$. In $\boldsymbol{B}$ and C, SST immunoreactivity (IR) is also shown. D, Expression of td-Tomato and CR IR in the SST/CR intersectional line. The area in the dashed box is enlarged in D1. $\boldsymbol{E}$, Expression of td-Tomato and CR IR in the SST/Calb intersectional line. The area in the dashed box is enlarged in E1.F, Left, The percentage of SST-immunopositive cells expressing td-Tomato in the SST/CR intersectional line across layers. Right, The percentage of td-Tomato cells that express CR in the SST/CR intersectional line across layers. G, Left, The percentage of SST-immunopositive cells expressing td-Tomato in the SST/Calb intersectional line across layers. Right, The percentage of td-Tomato cells that express Calb in the SST/Calb intersectional line across layers.

criterion on the on the amount of recovered axon in L1 but not in L2/3 would reveal the presence of two morphologically distinct Martinotti types without a bias due to axonal preservation in L1. We performed an unsupervised hierarchical cluster analysis using the relative axonal length and relative amount of nodes of the ascending axon in L1-L4. The first branching of the hierarchical dendrogram separates granular- from supragranular-targeting SST-INs. We defined the cells targeting the granular layer as nonMartinotti cells and those targeting supragranular layers as Martinotti cells (Muñoz et al., 2014; Fig. 4A). The second branching segregates SST-INs targeting mainly L2/3 from cells targeting mainly L1. SST-INs that showed a prominent innervation of L2/3, with less ascending axon reaching $\mathrm{L} 1$ were defined as fanning-out Martinotti cells (Muñoz et al., 2017). The third cluster consisted of cells that targeted mainly L1; we defined these cells as T-shaped Martinotti cells (Muñoz et al., 2017). We then trained a linear discriminant analysis classifier with the morphological parameters of these 17 cells and used it to classify the remaining 13 reconstructed cells. The resulting classification was in good agreement with the original visual identification using the distribution of axonal length and nodes of the ascending axon, and only one fanning-out Martinotti cell was misclassified as a T-shaped Martinotti.

Non-Martinotti cells are characterized by their specific innervation of L4. Indeed, cells belonging to this morphological group had $87.6 \pm 4 \%$ of their ascending axon, and $92 \pm 3 \%$ of their axonal nodes in L4 (Fig. 4 C, right). Non-Martinotti cells are also characterized by having a very small amount of axon in L1 $0.2 \pm$ $0.2 \%$ ) that does not branch in this layer. All non-Martinotti cells were recorded in the SST-cre mouse line (Fig. 4A).

Fanning-out Martinotti cells are characterized by targeting mainly L2/3, where these cells have $65.8 \pm 6 \%$ of their ascending axon, and $71 \pm 8 \%$ of their nodes. A smaller proportion (18 \pm $4 \%$ ) of the axon of these cells can reach the deeper half of L1, where it branches (Fig. 4C, left).

T-shaped Martinotti cells specifically target L1, where they have $50 \pm 3 \%$ of their ascending axonal length, and $56 \pm 4 \%$ of their ascending axonal nodes (Fig. 4C, center).

\section{Electrophysiological properties of morphologically identified SST interneurons}

Layer 5 SST-INs have been previously shown to have heterogeneous spiking properties in slice recordings (Kawaguchi and Kubota, 1997; Wang et al., 2004; Ma et al., 2006). In the mouse barrel cortex, L5 SST-INs have been shown to display three main types of firing pattern in response to membrane depolarization: L4-targeting neurons labeled in the X94 mouse line have a "quasifast spiking" firing pattern characterized by low-input resistance, fast AP kinetics and biphasic AHP, and stuttering APs trains (i.e., the AP train was randomly interrupted repetitively), whereas 
A B

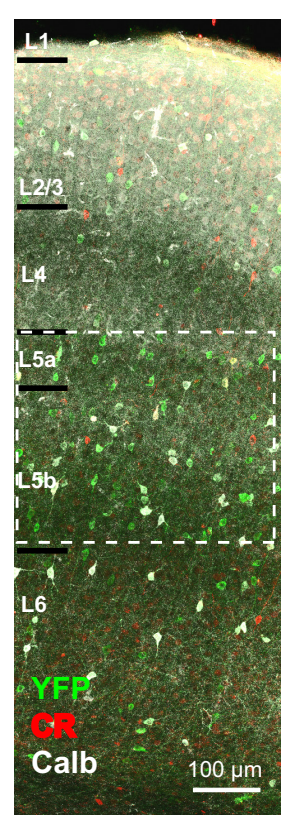

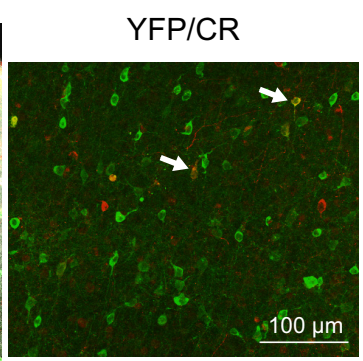

$\mathbf{E}$

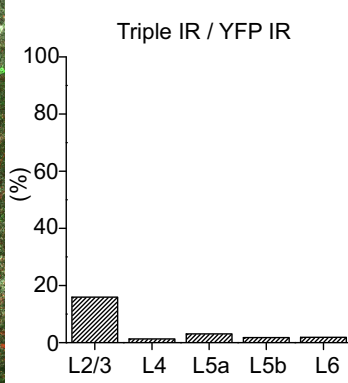

C

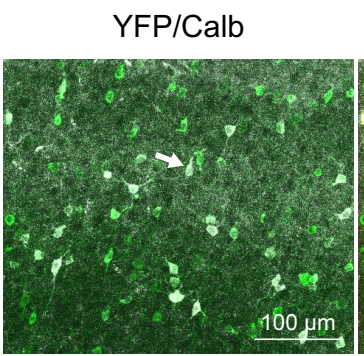

$\mathbf{F}$

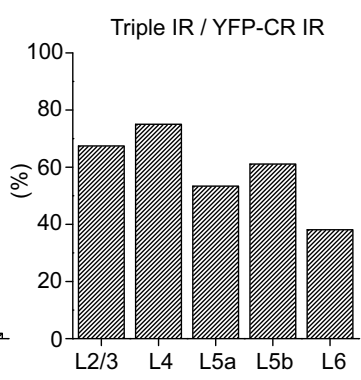

D

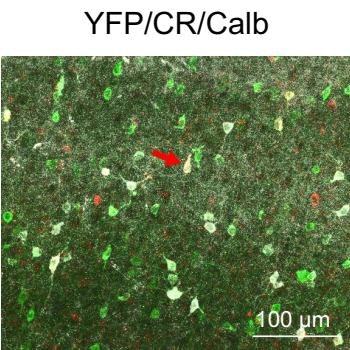

G

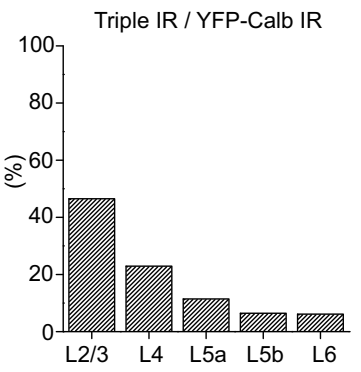

Figure 2. $\quad \boldsymbol{A}$, Confocal stack showing triple immunostaining for CR, Calb, and YFP in the SST-cre/YFP mouse. $\boldsymbol{B}-\boldsymbol{D}$, Enlarged view of the area delineated by the dashed box in $\boldsymbol{A}$, representing $\mathrm{L} 5$. White arrows indicate double-labeled cells. Red arrows indicate triple-labeled cells. B, Merged image of CR and YFP IR. C, Merged image of Calb and YFP IR. D, Merged image of CR, Calb, and YFP IR. E, Percentage of cells expressing CR, Calb, and YFP in the YFP IR population. F, Percentage of CR, Calb, and YFP-expressing cells in the YFP-CR IR population. G, Percentage of cells expressing CR, Calb, and YFP in the YFP-Calb IR population.

A

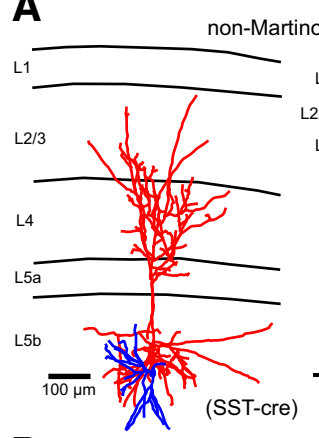

D

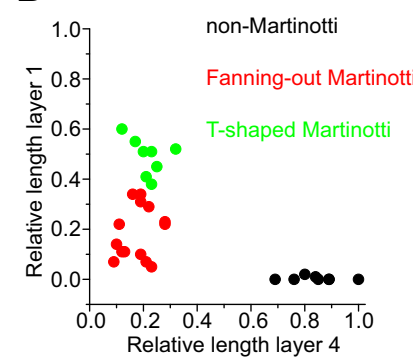

B

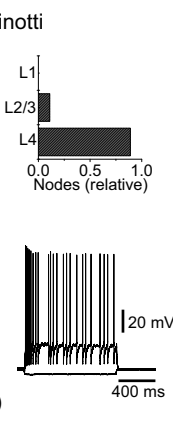

$\mathbf{E}$

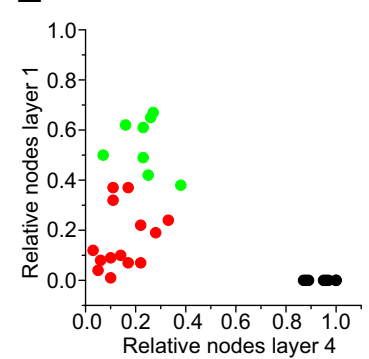

C

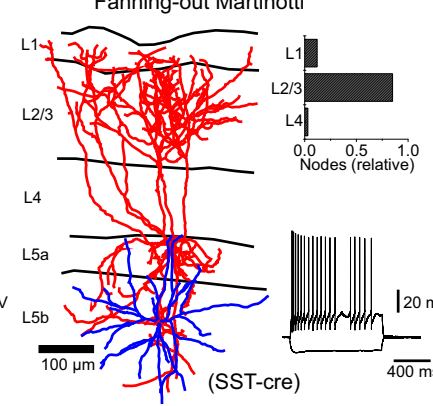

$\mathbf{F}$

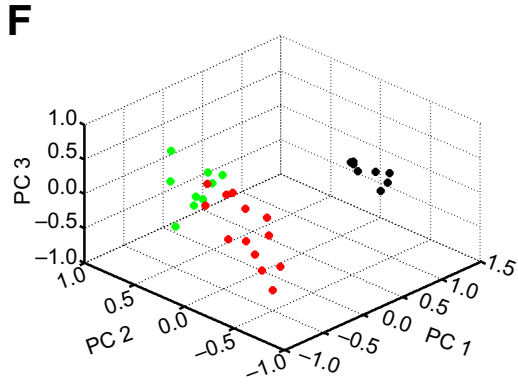

Figure 3. $\boldsymbol{A}-\boldsymbol{C}$, Reconstruction of a representative non-Martinotti cell $(\boldsymbol{A})$, fanning-out Martinotti cell ( $\boldsymbol{B})$, and a T-shaped Martinotti cell ( $\boldsymbol{C}$ ). Each reconstruction is accompanied on the right by the distribution of nodes relative to the total amount of nodes of the ascending axon in L1-L4, and by the firing pattern of each illustrated cell. Shown are traces during a hyperpolarizing and a suprathreshold depolarizing step. $\boldsymbol{D}$, Plot of the relative axonal length of the ascending axon in $L 1$ versus $L 4$ of the 30 reconstructed cells. $E$, Plot of the relative amount of nodes of the ascending axon in $L 1$ versus $L 4$ of the 30 reconstructed cells. $F, 3-D$ plot of the first three principal components of the principal component analysis using 16 morphological parameters of the axon of the 30 reconstructed cells (see Materials and Methods).

Martinotti neurons labeled in the X98 mouse line were shown to express either an adapting or a low-threshold spiking (LTS) firing pattern (Ma et al., 2006). To understand the relationship between the diversity of electrophysiological and morphological properties we analyzed 11 electrophysiological parameters that describe the firing properties of 29 of the 30 morphologically identified cells described above. We found that fanning-out and T-shaped Martinotti interneurons differed in their electrophysiological properties (Fig. $5 A, B$ ). The majority of fanning-out Martinotti cells showed an adapting firing pattern characterized by trains of APs that after an initial adaptation showed regular ISIs (Fig. 5A, left). Moreover, T-shaped Martinotti showed the lowest $F_{\text {max }}$, 


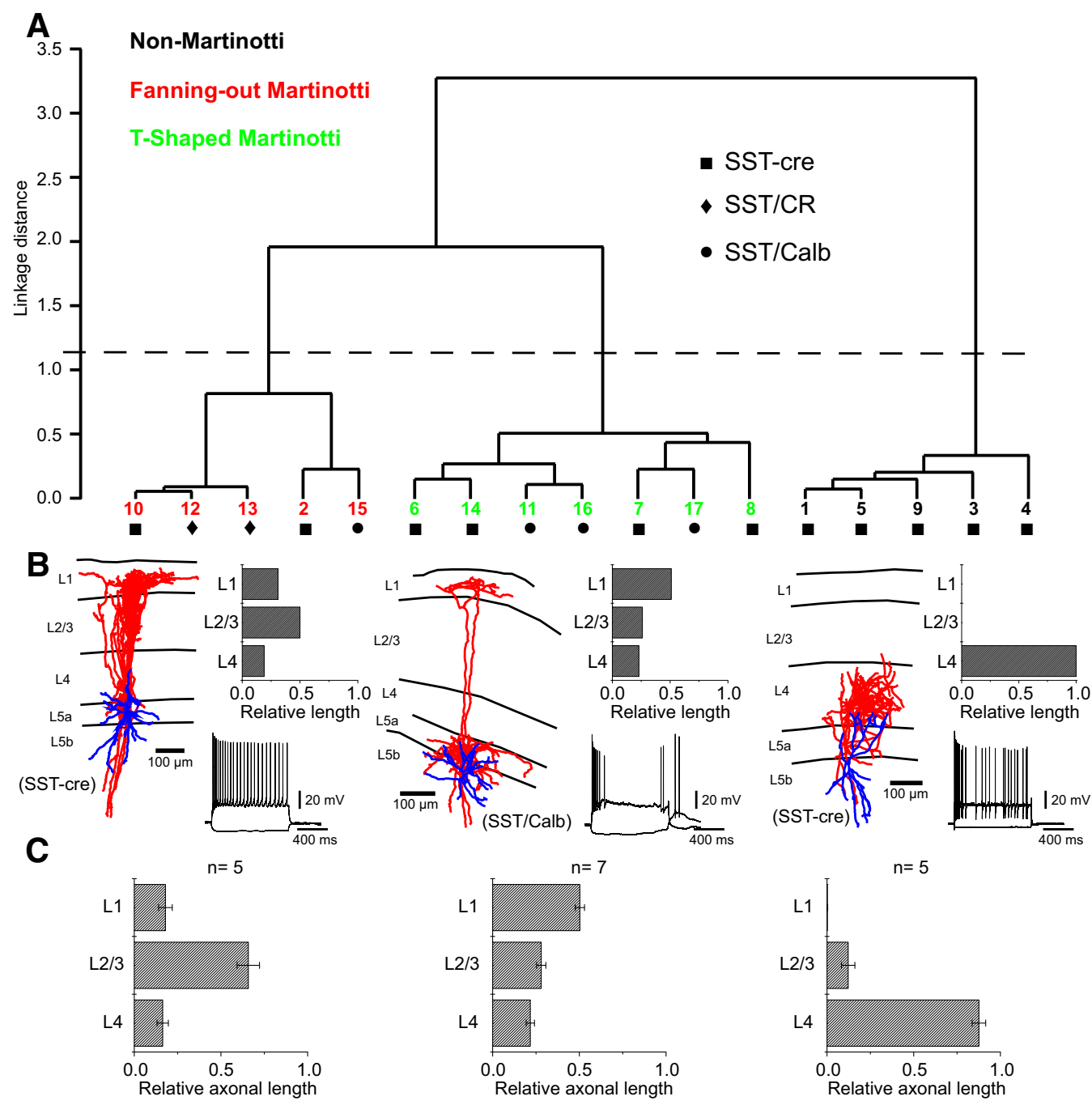

Figure 4. A, Dendrogram obtained from a hierarchical cluster analysis of 17 reconstructed SST cells. The dashed line represents the level at which the clusters were created according to the Thorndike method. Numbers on the $x$-axis represent the cells' ID color-coded according to the morphological group they were assigned to. B, Reconstructions of representative SST cells belonging to each morphological group. Each reconstruction is accompanied on the right by the distribution of relative axonal length of the ascending axon and the firing pattern for that cell. $C$, Plots showing the average distribution of the relative axonal length of the ascending axon for each morphological group.

likely due to their tendency to reach depolarization block early during the $f-I$ curve (Fig. $6 A$ ). Only 2 of 14 fanning-out cells showed rebound spiking (Fig. $5 C$, left). Fanning-out cells had a significantly higher $F_{\max }$, a more depolarized $V_{\text {threshold }}$ (Fig. 6C), and a smaller adaptation index compared with T-shaped Martinotti cells (Table 1; Fig. 6A-C). In summary, fanning-out Martinotti cells showed intermediate values in most electrophysiological parameters compared with the other two morphological groups. On the other hand, 7 of 8 T-shaped cells showed rebound spiking and a LTS firing pattern (Fig. $5 C$, center), showing a high IR, a low $\mathrm{AP}_{\text {threshold }}$, and a high adaptation index (Table 1; Fig. $6 A-C$ ).

We found that all the non-Martinotti showed stuttering $(n=$ 8), and 7 of 8 cells showed a fast biphasic AHP (Fig. $5 B$, right). Non-Martinotti cells showed several features of the quasi-fast spiking phenotype such as a lower IR, a shorter $\mathrm{AHP}_{\text {duration }}$, and a higher $F_{\max }$ compared with Martinotti cells (Table 1; Fig. $6 A-C$ ). We did not find a significant difference in the HW as previously reported for X94 cells (Ma et al., 2006), however, the rising phase of the AP was faster than that of Martinotti cells (Table 1). We did not find any significant correlation between the age of the animal and the electrophysiological properties analyzed here.
We took advantage of the good correlation between morphological and electrophysiological properties to estimate how the diversity of SST-INs is represented in the total population of SST-INs labeled in the three mouse lines used in this work. We first trained a linear discriminant classifier using six electrophysiological parameters that described the three types of firing patterns associated to 20 of the 30 morphologically identified cells (training group): IR, $\mathrm{AHP}_{\text {duration }}, \mathrm{AHP}_{\text {shape }}$, rebound spiking, $F_{\max }$, and $V_{\text {threshold. }}$. We then used the classifier to classify 76 neurons (of 96 total; test group, excluding cells used to build the classifier): 51 (of 65 total) in the SST-cre line, 16 (of 18 total) in the SST/CR line, and 9 (of 13 total) in the SST/Calb line. Among the 76 cells there were 21 morphologically preserved and visually identified cells that we used as control group for the classifier (10 reconstructed and 11 only visually identified). The classifier performed correctly for 19 of 21 cells using just six parameters. In the control group the classifier correctly identified all the nonMartinotti cells $(n=10), 7$ of 8 fanning-out Martinotti cells, and 2 of $3 \mathrm{~T}$-shaped cells. The two errors were a fanning-out misclassified as T-shaped likely because of a pronounced rebound spiking, although it had an adapting firing pattern, and a T-shaped 
A

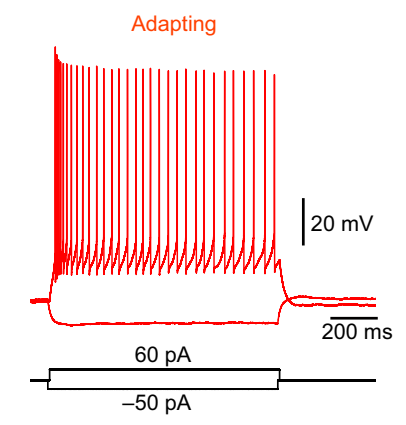

B

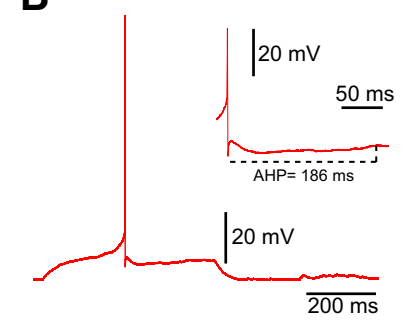

$16 \mathrm{pA}$
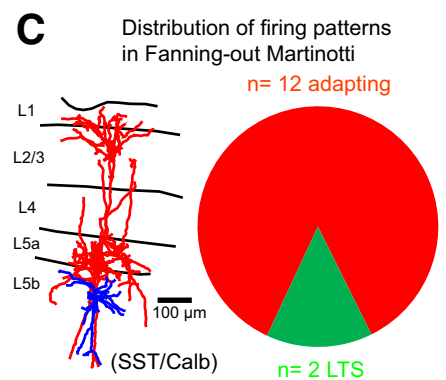

LTS
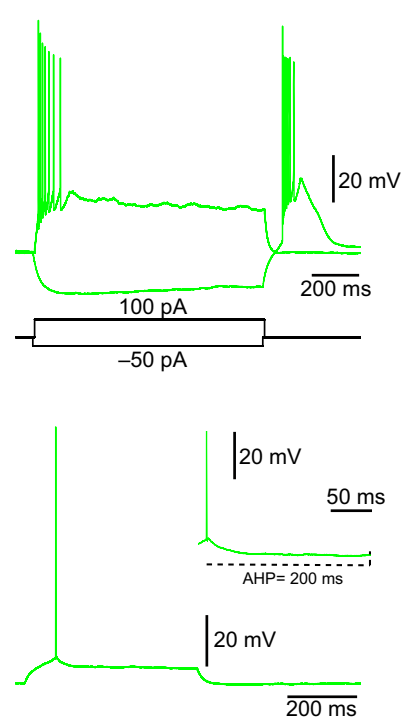

$30 \mathrm{pA}$

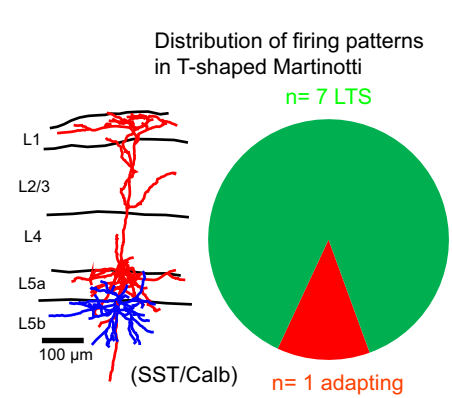

Quasi-fast spiking
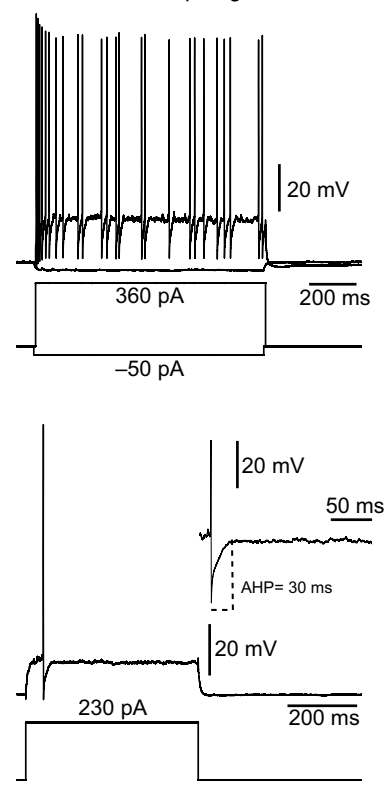

Distribution of firing patterns in non-Martinotti

$\mathrm{n}=8$ Quasi-fast spiking

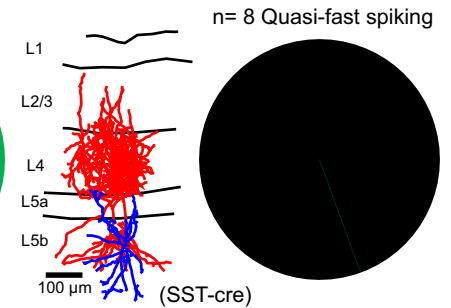

Figure 5. A, Representative voltage responses to hyperpolarizing and depolarizing current injections of fanning-out Martinotti, T-shaped Martinotti, and non-Martinotti cells. Injected current steps are shown below the voltage responses. $\boldsymbol{B}$, Representative voltage responses at rheobase current injections of fanning-out Martinotti, T-shaped Martinotti, and non-Martinotti cells. The injected current step is shown below the voltage response. Insets show AHP waveforms and report the respective duration (see Materials and Methods). C, Morphological reconstructions of the cells illustrated in $\boldsymbol{A}$ and $\boldsymbol{B}$. Pie charts show the number of cells with a specific firing pattern in each morphological group (red, fanning out Martinotti; green, T-shaped; black, non-Martinotti).

Martinotti misclassified as non-Martinotti although the firing pattern was clearly LTS. Given that no other similar error was found in the test group we concluded that the performance of the classifier was reliable. To increase the confidence for the T-shaped Martinotti group, we built a linear discriminant analysis using all 29 morphologically and electrophysiologically analyzed cells and classified the remaining 67 cells. We estimate that in the SST-cre mouse line, which labels virtually $100 \%$ of the SST-INs in the barrel cortex, fanning-out Martinotti cells represent $50.8 \%$ of labeled cells in L5, T-Shaped cells represent $13.8 \%$ of labeled cells, and non-Martinotti represent $35.4 \%$ of labeled cells (Fig. 6E, left). The intersectional mice used in the present work showed some specificity for morphological groups. The SST/CR mouse line targets mainly fanning-out Martinotti cells, that represented $88.9 \%$ of labeled cells, whereas none of the cells was classified as T-shaped Martinotti, and non-Martinotti cells $11.1 \%$ of labeled cells (one of which was morphologically confirmed; Fig. 6E, middle). The SST/Calb mouse line showed some enrichment for T-shaped Martinotti neurons that represented $61.5 \%$ of labeled cells (compared with $13.1 \%$ in the SSTCre line), whereas fanning-out Martinotti cells represented $30.8 \%$ of labeled cells, and non-Martinotti cells represented only $7.7 \%$ of labeled cells.

\section{Connectivity of morphological SST groups}

Cortical SST-INs are known to target the dendrites of pyramidal neurons (Wang et al., 2004; Silberberg and Markram, 2007). Layer 5 SST-INs have been shown to underlie frequencydependent disynaptic inhibition of thick tufted pyramidal cells (Silberberg and Markram, 2007). However the morphological diversity shown here suggests that different types of L5 SST-INs might have different postsynaptic partners and/or target different compartments of L5 pyramidal cells. To address this issue we performed dual and triple recordings of SST-INs and nearby pyramidal cells in L5. We tested a total of 39 pairs between L5 SST-INs and nearby pyramidal cells. We grouped the paired recordings according to the morphological identity of the SST-INs and obtained pyramidal cell pairs with 16 fanning-out Martinotti cells, 10 T-shaped Martinotti cells, and 13 non-Martinotti cells. We found that both types of Martinotti cells innervate L5 pyramidal neurons with similar connection probabilities (fanningout to pyramidal: $53.6 \%$, 9 of 16 ; T-shaped to pyramidal: $40 \%, 4$ of 10; Fig. $7 A-D$ ). We did not find any significant difference in the IPSC charge evoked by Martinotti cells onto pyramidal cells (fanning-out Martinotti: $965.6 \pm 204.5 \mathrm{nC}$; T-shaped Martinotti: $1232.6 \pm 262.3 \mathrm{nC} ; p=0.48$, two-sample $t$ test). We also quantified the putative synaptic contacts in connected pairs. We did 
A

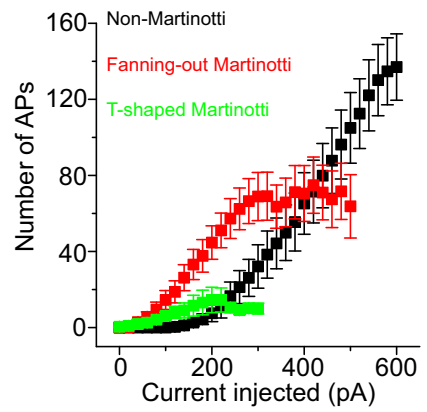

D

Fanning-out Martinotti

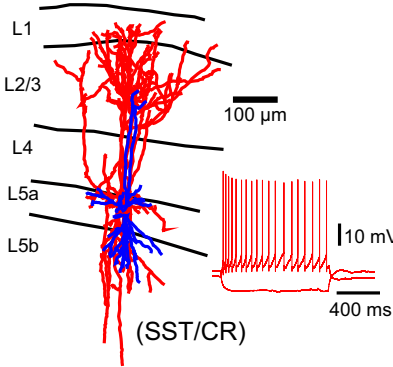

E

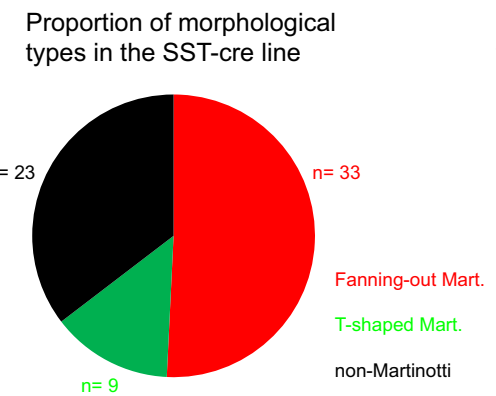

B

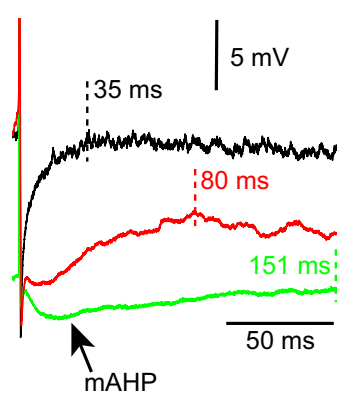

T-shaped Martinotti

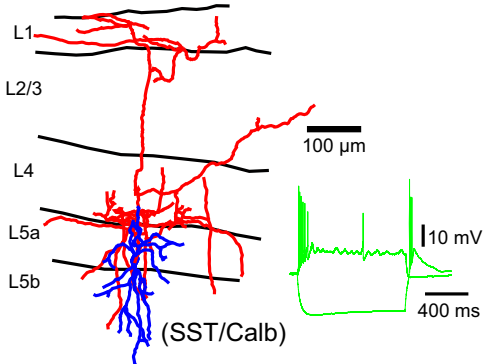

Proportion of morphological types in the SST/CR line

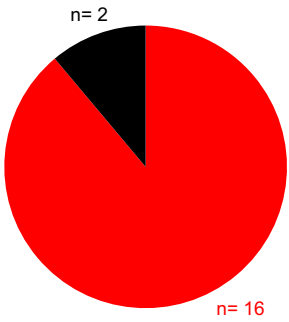

\section{C}

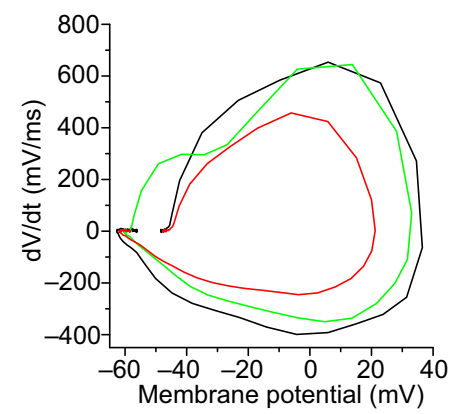

non-Martinotti

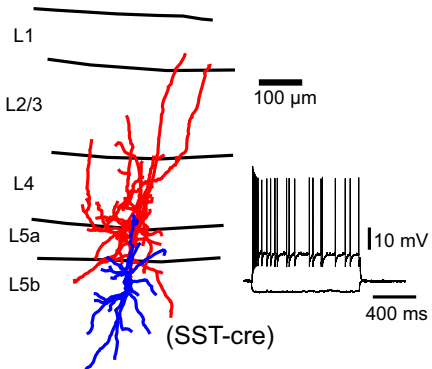

Proportion of morphological types in the SST/Calb line

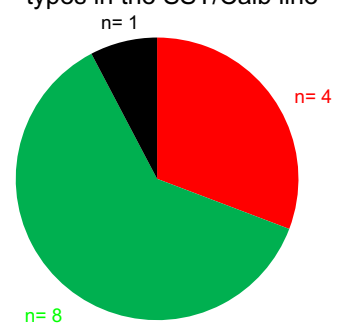

Figure 6. $\quad A$, Graph showing the $f-I$ curves of 29 morphologically identified SST-INs. B, Representative examples of the AHPs of a fanning-out Martinotti (red), a T-shaped Martinotti (green), and a non-Martinotti (black). The black arrow points to the mAHP. Dashed lines indicate the time point used to determine AHP duration. C, Graph showing the phase-plot of the cells illustrated in B. D, Three examples of correctly classified cells that were morphologically preserved and visually identified as fanning-out Martinotti (left), T-shaped Martinotti (middle), and non-Martinotti (right) cells. E, Pie charts showing the number of cells classified as fanning-out Martinotti, T-shaped Martinotti, or non-Martinotti cells in the three mouse lines used in the present study:SST-cre (left), SST/CR Flp/Cre (middle), and SST/Calb Flp/Cre (right).

Table 1. Electrophysiological properties of morphologically identified SST-INs

\begin{tabular}{|c|c|c|c|c|}
\hline Parameter & $\begin{array}{l}\text { Fanning-out } \\
\text { Martinotti, mean } \pm \text { SE } \\
n=14\end{array}$ & $\begin{array}{l}\text { T-shaped Martinotti, } \\
\text { mean } \pm S E \\
n=7\end{array}$ & $\begin{array}{l}\text { Non-Martinotti, } \\
\text { mean } \pm S E \\
n=8\end{array}$ & $\begin{array}{l}p \text { value } \\
\left(F_{(2,26)}\right)\end{array}$ \\
\hline$V_{\text {rest }}, \mathrm{mV}$ & $-63.6 \pm 2.6$ & $-63.3 \pm 3.3$ & $-63.9 \pm 2.1$ & $0.991(0.009)$ \\
\hline $\mathbb{I R}, \mathrm{M} \Omega$ & $195.3 \pm 28.1$ & $282.5 \pm 53$ & $83.2 \pm 4.5$ & $0.003(7.48)$ \\
\hline Sag & $0.93 \pm 0.02$ & $0.94 \pm 0.03$ & $0.96 \pm 0.01$ & $0.412(0.92)$ \\
\hline Rebound, APs & $1.1 \pm 0.7$ & $4.1 \pm 1.3$ & $0 \pm 0$ & $0.01(5.51)$ \\
\hline $\mathrm{AP}_{\text {thre }}, \mathrm{mV}$ & $-45.2 \pm 1.3$ & $-52.7 \pm 1.6$ & $-46.3 \pm 1.0$ & $0.003(7.3)$ \\
\hline $\mathrm{AP}_{\text {rise, }}, \mathrm{mV} / \mathrm{ms}$ & $507.8 \pm 25.1$ & $452.8 \pm 63.4$ & $628 \pm 49.3$ & $0.034(0.034)$ \\
\hline $\mathrm{HW}$, ms & $0.32 \pm 0.02$ & $0.39 \pm 0.05$ & $0.3 \pm 0.04$ & $0.171(1.89)$ \\
\hline $\mathrm{AHP}_{\mathrm{dur}}, \mathrm{ms}$ & $116.6 \pm 12.2$ & $134 \pm 19.5$ & $40.9 \pm 3.3$ & $2.158 \mathrm{E}-4(11.89)$ \\
\hline $\mathrm{AHP}_{\text {shape }}, \mathrm{mV}$ & $-1.7 \pm 0.3$ & $-1.1 \pm 0.4$ & $3.1 \pm 0.7$ & $1.5 \mathrm{E}-7(30.54)$ \\
\hline$F_{\max }, \mathrm{Hz}$ & $89.8 \pm 13.8$ & $29.9 \pm 15.3$ & $142.6 \pm 18.7$ & $7.545 \mathrm{E}-4(9.6)$ \\
\hline Adaptation & $0.69 \pm 0.05$ & $0.88 \pm 0.06$ & $0.76 \pm 0.03$ & $0.044(3.52)$ \\
\hline
\end{tabular}

Mean $\pm S E$ is reported for all electrophysiological parameters measured. The $p$ value of the $F$ statistics reports which parameters showed significantly different distribution of the means. Mean comparisons (Bonferroni): IR, T-shaped Martinotti versus non-Martinotti: $t_{(3.83)}, p=0.002$; Rebound, T-shaped Martinotti versus non-Martinotti: $t_{(3.19)}, p=0.011$, T-shaped Martinotti versus fanning-out Martinotti: $t_{(2.64)}, p=0.041$; AP thre, T-shaped Martinotti versus

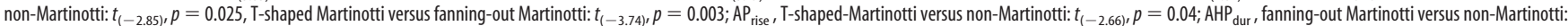

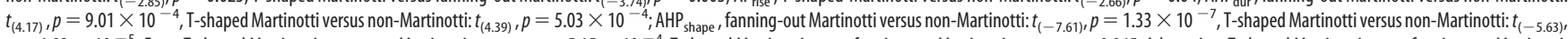
$p=1.93 \times 10^{-5} ; F_{\max }$, T-shaped Martinotti versus non-Martinotti: $t_{(-4.38)}, p=5.15 \times 10^{-4}$, T-shaped Martinotti versus fanning-out Martinotti: $t_{(-2.6)}, p=0.045 ;$ Adaptation, T-shaped Martinotti versus fanning-out Martinotti: $t_{(2.65)}, p=0.04$ 
A

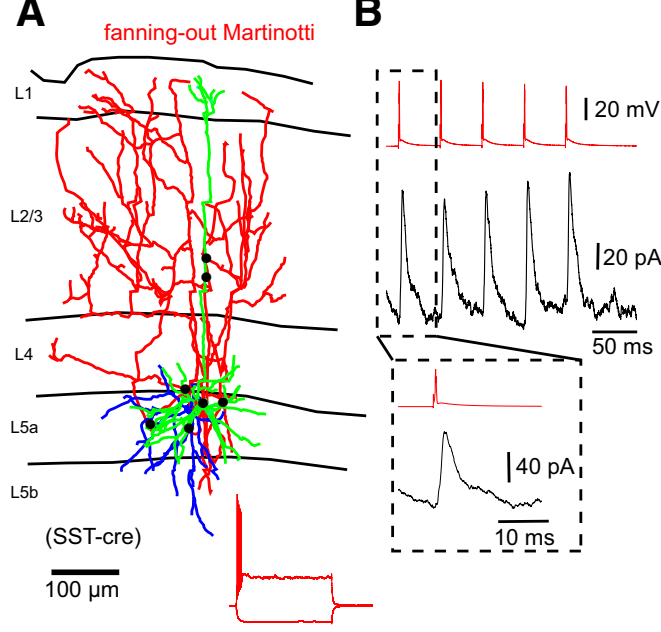

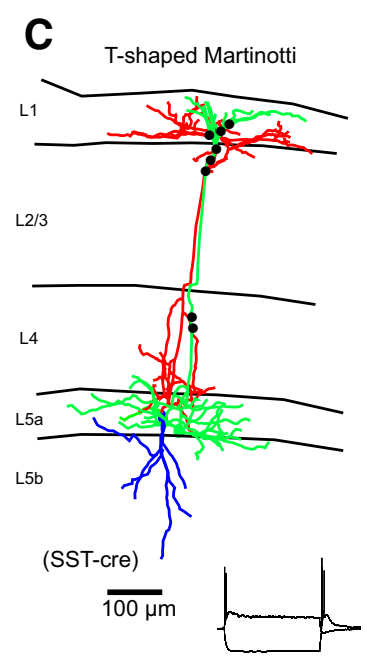

D

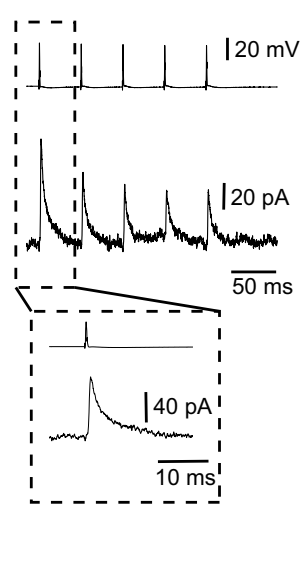

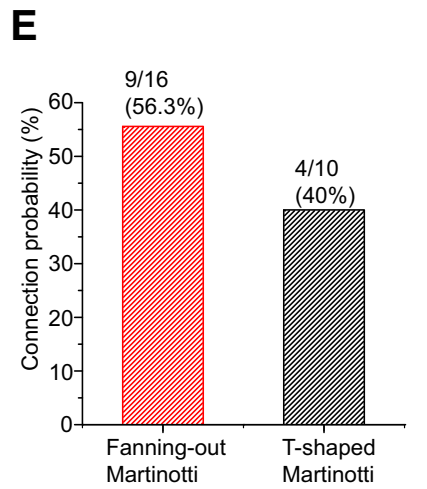
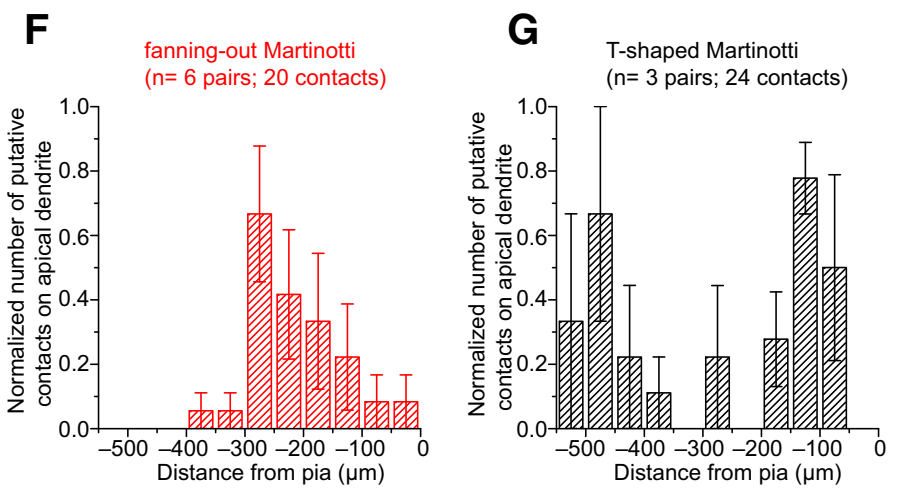

Figure 7. A, Morphological reconstruction of a connected pair between a fanning-out Martinotti cell (blue, dendrites; red, axon) and a L5 pyramidal neuron (green). Black filled circles represent putative synaptic contacts. Inset, The firing pattern of the fanning-out Martinotti cell. $\boldsymbol{B}$, IPSCs recorded on the pyramidal cell following stimulation the fanning-out Martinotti cell with a train of action potentials at $20 \mathrm{~Hz}$. Inset, The first IPSC at higher resolution. C, Morphological reconstruction of a connected pair between a T-shaped Martinotti cell (blue, dendrites; red, axon) and a L5 pyramidal cell (green). Black filled circles represent putative synaptic contacts. Inset, The firing pattern of the T-shaped Martinotti cell. D, IPSCs recorded from the pyramidal cell following stimulation of the T-shaped Martinotti cell with a train of action potentials at $20 \mathrm{~Hz}$. Inset, The first IPSC at higher resolution. E, Bar graph showing the connection probability for both types of Martinotti cell and L5 pyramidal neurons. $\boldsymbol{F}$, Histogram showing the distribution of the synaptic contacts of fanning-out Martinotti cells on the apical dendrites of $L 5$ pyramidal cells. $\mathbf{G}$, Histogram showing the distribution of the synaptic contacts of T-shaped Martinotti cells on the apical dendrite of $L 5$ pyramidal cells.

not find any significant difference in the amount of contacts in the basal or apical dendrites of pyramidal cells. Given the different axonal distribution across layers of the two types of Martinotti cells we hypothesized that they might target different compartments of the apical dendrites of L5 pyramidal cells. We measured the distance from the pia of the contact points located on the apical dendrites of L5 pyramidal neurons, and found that the distribution of contact points was significantly different between the two types of Martinotti cells ( $p=0.033$, two-sample Kolmogorov-Smirnov test; Fig. $7 F, G$ ). Indeed while fanning-out Martinotti cells had most of their synapses located in L2/3, between 150 and $300 \mu \mathrm{m}$ below the pia (Fig. 7 A, F), T-shaped Martinotti cells showed a bimodal distribution with a peak in L4 $(\sim 450 \mu \mathrm{m}$ below the pia) and a second peak in L1 and at the border between $\mathrm{L} 1$ and $\mathrm{L} 2 / 3$ (Fig. $7 C, G$ ).

The connectivity of non-Martinotti cells is unknown. We hypothesized that this group of SST-INs might inhibit the apical dendrites of L5 pyramidal cells in L4 and/or the dendrites of L4 neurons. Our dual and triple recordings showed that these cells are not connected to L5 pyramidal cells (0 of 13). We point out that for all the connections analyzed and presented in this study we had clear morphological evidence showing the apical dendrite of the pyramidal cells was intact and passed through the axonal domain of the SST-INs. Thus, we can exclude slicing artifacts as an explanation of the observed lack of connectivity of nonMartinotti cells and L5 pyramidal cells (Fig. $8 A, C$ ). The striking lack of connectivity between non-Martinotti and pyramidal cells, suggests that these cells might inhibit specifically neurons located in L4. Indeed we found a high connection probability of nonMartinotti cells with L4 spiny stellate cells $(83.3 \%, 5$ of 6$)$. All the putative contact points between non-Martinotti cells and L4 spiny stellate cells were located on dendrites (Fig. $8 C, G$ ).

\section{Discussion}

In the present study we addressed several important issues regarding the diversity of SST-INs in L5: (1) the correlation between morphological, electrophysiological and molecular features of SST-INs subtypes, (2) the relative abundance of each morphological type, and (3) the output connectivity of each morphological type with L5 PCs. Our study offers a complementary view to the recently described morphological diversity of SST-INs in vivo (Muñoz et al., 2017). We found in vitro the same morphological diversity observed by Muñoz et al. (2017) in vivo and were able to correlate morphological subtypes to the diverse firing patterns observed in the SST-INs population (Ma et al., 2006). We then used this correlation to infer the proportion of SST-INs belonging to each morphological group. The 
A

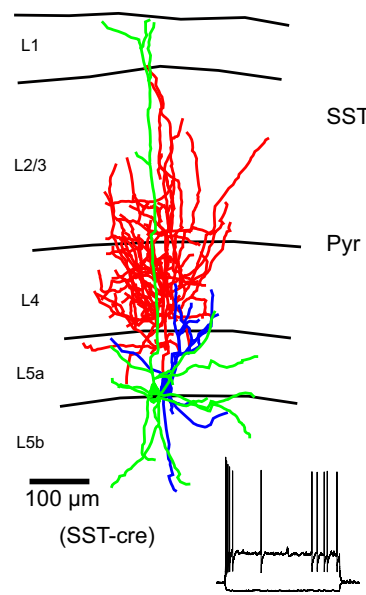

E

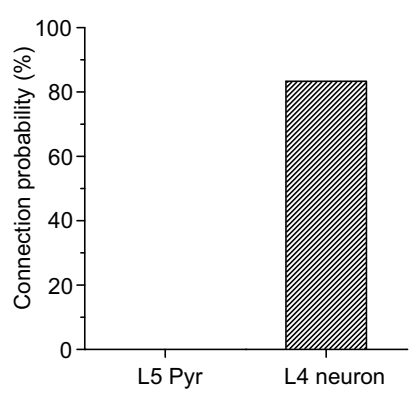

B

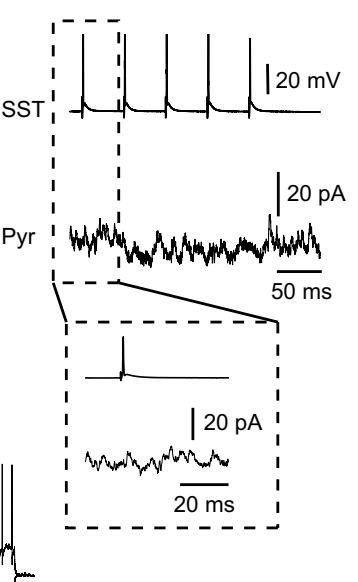

F

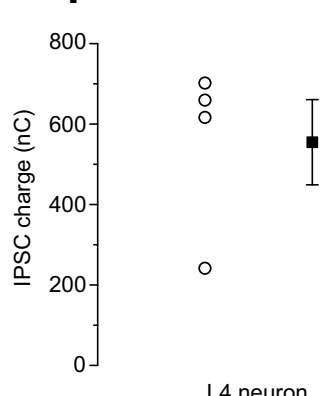

C

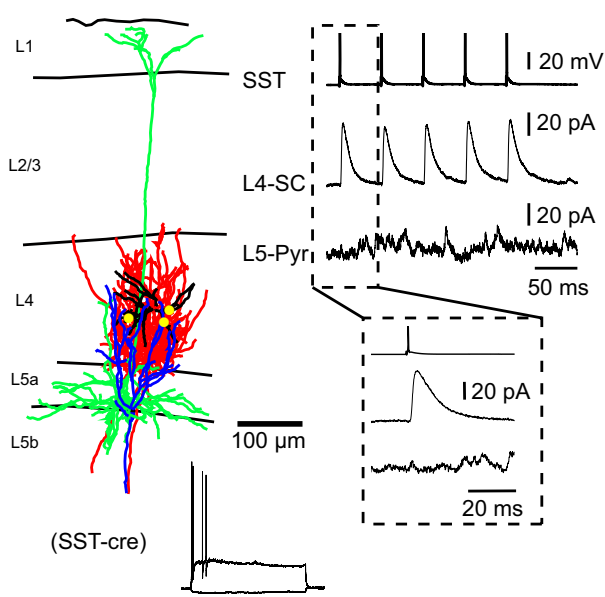

G

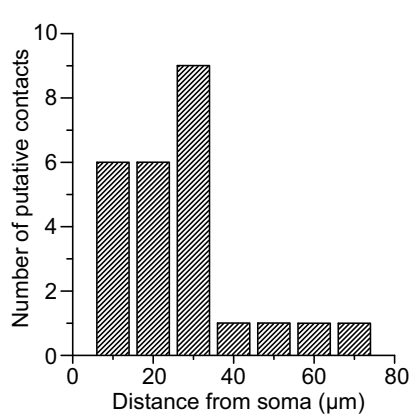

Figure 8. A, Morphological reconstruction of a non-connected pair between a non-Martinotti cell (blue, dendrites; red, axon) and a $L 5$ pyramidal cell (green). Inset, The firing pattern of the non-Martinotti cell. $\boldsymbol{B}$, A train of action potentials at $20 \mathrm{~Hz}$ in the $\mathrm{L} 5$ non-Martinotti cell did not evoke IPSCS on the $L 5$ pyramidal neuron. $\boldsymbol{C}$, Morphological reconstruction of a triple recording between a non-Martinotti cell (blue, dendrites; red, axon) a L5 pyramidal cell (green) and a L4 spiny stellate cell (black). Yellow filled circles represent putative synaptic contacts. Inset, The firing pattern of the non-Martinotti cell. $\boldsymbol{D}$, A train of action potentials at $20 \mathrm{~Hz}$ on the non-Martinotti cell did not evoke IPSCS on the L5 pyramidal neurons, but reliably evoked IPSCS on the L4 neuron. $\boldsymbol{E}$, Graph reporting the connection probability of paired recordings between $L 5$ non-Martinotti cells and $L 5$ pyramidal cells or $L 4$ stellate cells. $F$, Graph reporting the synaptic charge of $L 4 / 5$ paired recordings between a $L 5$ non-Martinotti cell and a $L 4$ stellate cell recorded in voltage-clamp. $\mathbf{G}$, Distribution of synaptic contacts on the dendrites of $L 4$ spiny stellate cells as a function of distance from the soma of the stellate cells.

same approach allowed us to characterize the SST-INs population in two intersectional mouse lines. We found that the SST/CR line is highly enriched in fanning-out Martinotti cells, while the SST/Calb line is enriched in T-shaped Martinotti cells. Last, using paired recordings we show that morphologically distinct SST-INs belong to different microcircuits in the barrel cortex.

\section{Diversity of cortical SST-INs}

SST-INs are a very heterogeneous group of cortical GABAergic neurons that vary in morphological, electrophysiological, and molecular features (Tremblay et al., 2016). Morphologically, SST-INs can be divided in two broad classes: Martinotti cells having an ascending axon that reaches L1 and are located mainly in supragranular and infragranular layers; and non-Martinotti cells that are located in L4 and L5b and specifically target L4 (Ma et al., 2006). Recently this morphological diversity was confirmed in vivo (Muñoz et al., 2014, 2017). Moreover, Muñoz et al. (2017) showed that SST-INs belonging to a specific morphological group showed specific patterns of activity during different behavioral states. Furthermore, Muñoz et al. (2017) identified additional morphological diversity within the L5 Martinotti cell group. They observed that SST-INs with broad axonal branching in L2/3 (and named fanning-out Martinotti cells) were activated during whisking, whereas SST-INs that mainly targeted L1 (T- shaped Martinotti cells) were silenced during whisking (Muñoz et al., 2017). In the present study we show that the morphological diversity of SST-INs in L5 correlates with their spike properties and repetitive firing characteristics. We found that nonMartinotti cells showed a "quasi-fast spiking" phenotype, similar to that described in X94 cells (Ma et al., 2006). Within the Martinotti group, fanning-out Martinotti cells showed an adapting type of firing pattern reminiscent of the GIN SST-INs, and T-shaped Martinotti cells showed an LTS firing pattern similar to that observed in some X98 cells (Ma et al., 2006).

The relative abundance of morphologically defined SST-INs is still unclear. The markers currently available to segregate SST-IN subtypes are insufficient for this purpose. For example, whereas the X94 line, a transgenic mouse line expressing GFP under control of a GAD promoter, selectively targets non-Martinotti cells in L4 and L5b (Ma et al., 2006), only 40\% of the L4 population of non-Martinotti cells express GFP. Furthermore, no differences were detected between the L4 SST cells expressing GFP and those that did not (Xu et al., 2013). In this study, we used the firing properties of morphologically identified cells to build a linear discriminant classifier to infer the proportion of cells belonging to each morphological class in L5. We found that the majority (62.3\%) of L5 SST-INs belong to the Martinotti group. Specifically, $49.2 \%$ were classified as fanning-out and $13.1 \%$ were classified as 
T-shaped. Moreover we found that a substantial proportion (37.7\%) of L5 SST-INs was classified as non-Martinotti cells. We used the same classifier on the dataset obtained from two intersectional lines and found that the SST/CR mouse is enriched in fanning-out Martinotti cells, whereas the SST/Calb is enriched in T-shaped Martinotti cells. These results highlight the promise of correlating morphological, electrophysiological, and molecular properties to obtain tools to target and manipulate functionally relevant SST subgroups (Fenno et al., 2014; He et al., 2016). Additional markers and understanding of their correlation with morphological subtypes are still needed to develop more specific tools. Indeed, neither calretinin nor calbindin are optimal molecular markers for fanning-out and T-shaped Martinotti respectively. The SST/CR mouse line labels only part of the fanning-out population in L5, and the SST/Calb labels a population that is still very heterogeneous. An interesting approach might be to exploit the combination of patch-clamp recordings with single cell sequencing techniques to obtain more specific molecular markers in morphologically and/or electrophysiologically identified cells (Cadwell et al., 2016; Fuzik et al., 2016).

The electrophysiological properties that define the three groups of SST-INs described in L5 seem to be differentially distributed across layers. Indeed L2/3 SST-INs show adapting firing patterns resembling that of L5 fanning-out Martinotti (Halabisky et al., 2006; Ma et al., 2006; Xu et al., 2013). L2/3 SST-INs have been shown to be Martinotti cells targeting L1 (Ma et al., 2006; Dumitriu et al., 2007; Xu et al., 2013; Muñoz et al., 2017). On the other hand, L4 SST-INs have been shown to be quasi-fast spiking L4 targeting non-Martinotti cells (Ma et al., 2006; Xu et al., 2013; Muñoz et al., 2017). However, L5 non-Martinotti tend to show a higher input resistance and seem to be less stuttering (Ma et al., 2006). L6 SST-INs have not been characterized yet. It is likely that one or both types of Martinotti cells are also present in this layer (Ma et al., 2006; Muñoz et al., 2017). Long-range projecting nNos-expressing SST-INs have been described in L6 as well (He et al., 2016).

A wide morphological diversity has been described in other GABAergic interneuron groups, such as VIP-INs (bipolar and multipolar) and PV-INs (basket, chandelier, translaminar; DeFelipe et al., 1989; Buchanan et al., 2012; Bortone et al., 2014; Prönneke et al., 2015; He et al., 2016). This morphological diversity often correlates with electrophysiological and molecular diversity, but a clear picture of this correlation is still missing. The combination of well characterized intersectional mouse lines with single-cell sequencing seems a very promising approach to understand the molecular underpinnings of the morphological diversity of molecularly defined GABAergic interneurons (Paul et al., 2017).

\section{Connectivity of SST-INs subtypes}

SST-INs provide frequency-dependent feedback inhibition to pyramidal cells in supragranular and infragranular layers (Kapfer et al., 2007; Silberberg and Markram, 2007). This form of inhibition is thought to underlie surround inhibition, and gammaband synchronization in L2/3 of the visual cortex (Adesnik et al., 2012; Veit et al., 2017). In the neocortical L5, computational and experimental work has shown that Martinotti cells can synchronize pyramidal cells through distal inhibition ( $\mathrm{Li}$ et al., 2013; Hilscher et al., 2017). In the barrel cortex, L5 contains at least three morphological subtypes of SST-INs (Muñoz et al., 2017; present study). These morphological subtypes target different layers of the cortical column (Fig. 4) and are active during different behavioral states (Muñoz et al., 2017).
We showed that both types of L5 Martinotti cells contact nearby pyramidal neurons. As expected from their axonal distribution, T-shaped Martinotti form synapses onto tufts in L1, whereas fanning-out Martinotti target the apical dendrites in L2/3 (Figs. 4, 7). This selective targeting might have important functional consequences in gating different types of inputs impinging on different dendritic compartments, during different behavioral states (Petreanu et al., 2009; Muñoz et al., 2017). Indeed, T-shaped and fanning-out Martinotti cells may control $\mathrm{Ca}^{2+}$ electrogenesis in different dendritic compartments, differentially contributing to sensory perception (Murayama et al., 2009). Interestingly, T-shaped Martinotti cells seem to be the sole SST-IN subtype to provide dendritic inhibition to the apical dendrite of L5 PCs in L4, where these cells receive sensory inputs from the ventral posteromedial thalamic nucleus (Petreanu et al., 2009). Because T-shaped cells are silent during whisking, the connectivity shown here suggests that these cells allow the apical dendrites of L5 PCs to integrate bottom-up sensory information in L4 with top-down feedback projections impinging onto the apical tufts in L1 during whisking. It still remains to be addressed whether the two types of Martinotti cells show different connectivity with L2/3 neurons.

The output connectivity of L5 non-Martinotti cells was previously unknown. These cells, together with L4 SST-INs, contribute the majority of SST-INs axons in L4, suggesting a strong control over ascending sensory inputs. Here we show that L5 non-Martinotti cells specifically target L4 neurons and avoid L5 pyramidal cells. Using the X94 mouse line it was shown that L5 non-Martinotti cells receive thalamic input from the ventral posteromedial nucleus and that this input can shift thalamic feedforward inhibition from perisomatic to dendritic (Tan et al., 2008; $\mathrm{Hu}$ and Agmon, 2016). It will be of interest to understand the connectivity of L5 non-Martinotti cells with both local and L4 interneurons. Indeed L4 non-Martinotti cells have been suggested to disinhibit L4 excitatory cells by primarily targeting L4 parvalbumin interneurons (Xu et al., 2013).

In conclusion, we provide evidence that morphologically diverse L5 SST-INs participate in different microcircuits in the mouse barrel cortex. The actions of different SST-INs subtypes are likely to have important computational roles in sensory perception during different behavioral states.

Note Added in Proof: The first and last name of the first author were switched in the Early Release version of this article published January 11, 2018. The first author's name has now been corrected.

\section{References}

Adesnik H, Bruns W, Taniguchi H, Huang ZJ, Scanziani M (2012) A neural circuit for spatial summation in visual cortex. Nature 490:226-231. CrossRef Medline

Blasdel GG, Lund JS (1983) Termination of afferent axons in macaque striate cortex. J Neurosci 3:1389-1413. Medline

Bortone DS, Olsen SR, Scanziani M (2014) Translaminar inhibitory cells recruited by layer 6 corticothalamic neurons suppress visual cortex. Neuron 82:474-485. CrossRef Medline

Buchanan KA, Blackman AV, Moreau AW, Elgar D, Costa RP, Lalanne T, Tudor Jones AA, Oyrer J, Sjöström PJ (2012) Target-specific expression of presynaptic NMDA receptors in neocortical microcircuits. Neuron 75:451-466. CrossRef Medline

Cadwell CR, Palasantza A, Jiang X, Berens P, Deng Q, Yilmaz M, Reimer J, Shen S, Bethge M, Tolias KF, Sandberg R, Tolias AS (2016) Electrophysiological, transcriptomic and morphologic profiling of single neurons using patch-seq. Nat Biotechnol 34:199-203. CrossRef Medline

DeFelipe J, Hendry SH, Jones EG (1989) Visualization of chandelier cell axons by parvalbumin immunoreactivity in monkey cerebral cortex. Proc Natl Acad Sci U S A 86:2093-2097. CrossRef Medline

Dumitriu D, Cossart R, Huang J, Yuste R (2007) Correlation between ax- 
onal morphologies and synaptic input kinetics of interneurons from mouse visual cortex. Cereb Cortex 17:81-91. CrossRef Medline

Fenno LE, Mattis J, Ramakrishnan C, Hyun M, Lee SY, He M, Tucciarone J, Selimbeyoglu A, Berndt A, Grosenick L, Zalocusky KA, Bernstein H, Swanson H, Perry C, Diester I, Boyce FM, Bass CE, Neve R, Huang ZJ, Deisseroth K (2014) Targeting cells with single vectors using multiplefeature boolean logic. Nat Methods 11:763-772. CrossRef Medline

Florence SL, Casagrande VA (1987) Organization of individual afferent axons in layer IV of striate cortex in a primate. J Neurosci 7:3850-3868. Medline

Freund TF, Martin KA, Soltesz I, Somogyi P, Whitteridge D (1989) Arborisation pattern and postsynaptic targets of physiologically identified thalamocortical afferents in striate cortex of the macaque monkey. J Comp Neurol 289:315-336. CrossRef Medline

Fuzik J, Zeisel A, Máté Z, Calvigioni D, Yanagawa Y, Szabó G, Linnarsson S, Harkany T (2016) Integration of electrophysiological recordings with single-cell RNA-seq data identifies neuronal subtypes. Nat Biotechnol 34:175-183. CrossRef Medline

Halabisky B, Shen F, Huguenard JR, Prince DA (2006) Electrophysiological classification of somatostatin-positive interneurons in mouse sensorimotor cortex. J Neurophysiol 96:834-845. CrossRef Medline

He M, Tucciarone J, Lee S, Nigro MJ, Kim Y, Levine JM, Kelly SM, Krugikov I, Wu P, Chen Y, Gong L, Hou Y, Osten P, Rudy B, Huang ZJ (2016) Strategies and tools for combinatorial targeting of GABAergic neurons in mouse cerebral cortex. Neuron 91:1228-1243. CrossRef Medline

Hilscher MM, Leão RN, Edwards SJ, Leão KE, Kullander K (2017) Chrna2Martinotti cells synchronize layer 5 type A pyramidal cells via rebound excitation. PLoS Biol 15:e2001392. CrossRef Medline

Hu H, Agmon A (2016) Differential excitation of distally versus proximally targeting cortical interneurons by unitary thalamocortical bursts. J Neurosci 36:6906-6916. CrossRef Medline

Kapfer C, Glickfeld LL, Atallah BV, Scanziani M (2007) Supralinear increase of recurrent inhibition during sparse activity in the somatosensory cortex. Nat Neurosci 10:743-753. CrossRef Medline

Kawaguchi Y, Kubota Y (1997) GABAergic cell subtypes and their synaptic connections in rat frontal cortex. Cereb Cortex 7:476-486. CrossRef Medline

Lee LJ, Iwasato T, Itohara S, Erzurumlu RS (2005) Exuberant thalamocortical axon arborization in cortex-specific NMDAR1 knockout mice. J Comp Neurol 485:280-292. CrossRef Medline

Li X, Morita K, Robinson HP, Small M (2013) Control of layer 5 pyramidal cell spiking by oscillatory inhibition in the distal apical dendrites: a computational modeling study. J Neurophysiol 109:2739-2756. CrossRef Medline

Ma Y, Hu H, Berrebi AS, Mathers PH, Agmon A (2006) Distinct subtypes of somatostatin-containing neocortical interneurons revealed in transgenic mice. J Neurosci 26:5069-5082. CrossRef Medline
Martinotti C (1889) Contributo allo studio della corteccia cerebrale, ed all'origine centrale dei nervi. Ann Freniatr Sci Affini 1:14-381.

Muñoz W, Tremblay R, Rudy B (2014) Channelrhodopsin-assisted patching: in vivo recording of genetically and morphologically identified neurons throughout the brain. Cell Rep 9:2304-2316. CrossRef Medline

Muñoz W, Tremblay R, Levenstein D, Rudy B (2017) Layer-specific modulation of neocortical dendritic inhibition during active wakefulness. Science 355:954-959. CrossRef Medline

Murayama M, Pérez-Garci E, Nevian T, Bock T, Senn W, Larkum ME (2009) Dendritic encoding of sensory stimuli controlled by deep cortical interneurons. Nature 457:1137-1141. CrossRef Medline

Paul A, Crow M, Raudales R, He M, Gillis J, Huang ZJ (2017) Transcriptional architecture of synaptic communication delineates GABAergic neuron identity. Cell 171:522-539.e20. CrossRef Medline

Petreanu L, Mao T, Sternson SM, Svoboda K (2009) The subcellular organization of neocortical excitatory connections. Nature 457:1142-1145. CrossRef Medline

Prönneke A, Scheuer B, Wagener RJ, Möck M, Witte M, Staiger JF (2015) Characterizing VIP neurons in the barrel cortex of VIPcre/tdTomato mice reveals layer-specific differences. Cereb Cortex 25:4854-4868. CrossRef Medline

Ramon y Cajal S (1891) Sur la structure de l'ecorce cerebrale de quelques mammiferes. Cellule 7:3-54.

Silberberg G, Markram H (2007) Disynaptic inhibition between neocortical pyramidal cells mediated by Martinotti cells. Neuron 53:735-746. CrossRef Medline

Tan Z, Hu H, Huang ZJ, Agmon A (2008) Robust but delayed thalamocortical activation of dendritic-targeting inhibitory interneurons. Proc Natl Acad Sci U S A 105:2187-2192. CrossRef Medline

Tremblay R, Lee S, Rudy B (2016) GABAergic interneurons in the neocortex: from cellular properties to circuits. Neuron 91:260-292. CrossRef Medline

Veit J, Hakim R, Jadi MP, Sejnowski TJ, Adesnik H (2017) Cortical gamma band synchronization through somatostatin interneurons. Nat Neurosci 20:951-959. CrossRef Medline

Wang Y, Toledo-Rodriguez M, Gupta A, Wu C, Silberberg G, Luo J, Markram H (2004) Anatomical, physiological and molecular properties of Martinotti cells in the somatosensory cortex of the juvenile rat. J Physiol 561: 65-90. CrossRef Medline

Xu H, Jeong HY, Tremblay R, Rudy B (2013) Neocortical somatostatinexpressing GABAergic interneurons disinhibit the thalamorecipient layer 4. Neuron 77:155-167. CrossRef Medline

Xu X, Roby KD, Callaway EM (2010) Immunochemical characterization of inhibitory mouse cortical neurons: three chemically distinct classes of inhibitory cells. J Comp Neurol 518:389-404. CrossRef Medline

Yamada A, Uesaka N, Hayano Y, Tabata T, Kano M, Yamamoto N (2010) Role of pre- and postsynaptic activity in thalamocortical axon branching. Proc Natl Acad Sci U S A 107:7562-7567. CrossRef Medline 